\title{
Toward a new autoantibody diagnostic orthodoxy: understanding the bad, good and indifferent
}

\author{
Marvin J. Fritzler
}

Received: 4 March 2012/ Accepted: 7 March 2012/Published online: 21 March 2012

(C) Springer-Verlag 2012

\begin{abstract}
Rapid advances in diagnostic technologies used to detect autoantibodies have made it difficult for even the most modern laboratory to keep abreast of the changing approaches and platforms, not to mention the clinicians who are hard pressed to keep abreast of evolving diagnostic paradigms attended by these newer techniques. While autoantibody testing is traditionally considered to be primarily serving the realm of diagnostic medicine, there is little doubt that autoantibodies are also being recognized as an approach to providing prognostic and therapeutic information. Accordingly, along with related proteomics, genomics and metabolomics, it is taking on increasing importance in the realm of personalized medicine. In today's world of autoantibody diagnostics, overarching concerns about false-negative and false-positive autoantibodies tests cannot be summarily dismissed by citing pros or cons of any one technology or diagnostic platform, but often point to persisting gaps in our knowledge about, and understanding of, the origin and roles of autoantibodies. Before we can hope to completely understand the enigmas that attend the results of autoantibody diagnostic tests, perhaps it is time to step back and re-examine longaccepted paradigms and beliefs. This review will address some of the issues that impact on autoantibody detection technologies and some of the considerations and issues that will attend a new orthodoxy of autoantibody diagnostics. These issues will be addressed in the context of "bad" (pathogenic), "good" (protective) or "indifferent" (no apparent role in disease) autoantibodies.
\end{abstract}

M. J. Fritzler ( $\square)$

Faculty of Medicine, University of Calgary,

3330 Hospital Dr. NW,

Calgary, AB T2N 4N1, Canada

e-mail: fritzler@ucalgary.ca
Keywords Autoantibodies - Autoantigens ·

Diagnostics · Anti-nuclear antibodies

Overview: autoantibody detection in systemic autoimmune rheumatic diseases

The history of autoantibodies (aab) dates back more than a century to Ehrlich's description of "horror autotoxicus" [1] and subsequent observations that sera from syphilis, systemic lupus erythematosus and other inflammatory conditions reacted with Treponema-related components, including cardiolipin, in the Wasserman and the subsequent Venereal Diseases Research Laboratory (VDRL) tests [2-5]. Despite this long history, advances in the detection of antibodies were slow or spotty for about 50 years (i.e., 1900-1950) and was largely relegated to infectious diseases and, to a certain extent, organ-specific autoimmune diseases [6], but then achieved prominence in the following 50 years (i.e., 1950-2000). The tremendous surge in aab testing dates to the seminal observation of the lupus erythematosus (LE) cell phenomenon by Hargreaves and his colleagues [7] and then the development and wide use of the LE cell test [8]. With the notion that aab in human sera and other biological fluids could have diagnostic value beyond the realm of infectious diseases, a number of techniques such as immunofluorescence, immunodiffusion, hemagglutination and complement fixation were developed and refined in the following 20 years (reviewed in [9]). Indirect immunofluorescence (IIF), first described by Coons, Kaplan and Weller in the early 1950s [10, 11] almost stands alone as one of those early techniques that has stood the test of time as an important screening test in the diagnosis of systemic autoimmune rheumatic (SARD) and other diseases (reviewed in [12]). 
While the first IIF protocols utilized a variety of substrates, cryopreserved sections of rodent organs became the mainstay for $\sim 20$ years [13-16] until the mid-1970s, when it was discovered that human tissue culture cells, such as HeLa and HEp-2, derived from various malignancies were superior to organ sections primarily because they were easier to produce in large numbers, had larger nuclei (of value at a time when nucleolar antigens were a particular focus) and expressed novel antigens in various stages of the cell cycle [17, 18]. In retrospect, it is curious that carcinoma cell lines were chosen as the substrate of choice and, for reasons that are not clear, the development of IIF techniques for SARD diagnostics eventually relied almost entirely on HEp-2 rather than HeLa cells: this despite subsequent evidence that some presumed HEp-2 cell lines may actually be HeLa cells anyway $[19,20]$. In any event, the adoption of HEp- 2 cell substrates led to what might be regarded the "golden age" of aab detection in SARD. The increased sensitivity of IIF techniques on HEp-2 cells and a plethora of HEp-2 based IIF diagnostic kits became an issue in interpreting IIF anti-nuclear antibody results. It took almost a decade to finally appreciate that the serum dilutions used to detect aab on rodent cryosections were not appropriate on HEp-2 substrates. This led to a study by a Serology Subcommittee of the International Union of Immunology Societies/World Health Organization/Arthritis Foundation (IUIS/WHO/AF) who recommended a serum screening dilution of $1 / 160$ to achieve a balance of sensitivity and specificity in the serological diagnosis and classification of adult SARD [21]. In retrospect, it is obvious that this advice was not widely heeded, or perhaps accepted, because to this day many laboratories still screen at dilutions that they feel provide the appropriate balance of sensitivity/specificity.

One of the unanticipated advantages of IIF on HEp-2 cells was the ability to identify target autoantigens that were weakly expressed, selectively expressed or not expressed at all in differentiated organs such as rodent liver or kidney. This led to a virtual explosion of publications identifying novel antigen targets that had hitherto not been appreciated and to the point where there are now over 150 aab described in systemic lupus erythematosus (SLE) [22, 23] and over 30 in scleroderma [24]. These included anti-proliferating cell nuclear antigen (PCNA) and other cell cycle related targets (reviewed in [25]), anti-centromere (reviewed in [26]), and a number of targets in nucleoli (reviewed in [27, 28]), nuclear envelope [29] and cytoplasm (reviewed in [30]). The use of IIF to identify these novel targets was fortuitous because, in parallel, new techniques in cell and molecular biology such as immunoprecipitation, immunoblotting, expression cloning and rapid and economical DNA sequencing opened up the entire field of molecular biology that facilitated the identification of the more precise targets of human aab and in many cases completely novel proteins or nucleic acids that were unknown to basic cell and molecular biologists [31].

The tremendous strides in identifying the molecular targets of human aab quickly led to the next iteration of diagnostic technologies that used the molecular information to design, develop and market antigen-specific immunoassays using novel platforms that included enzyme-linked immunoassays (ELISA) [32-34], dot blots [35, 36], line immunoassays (LIA) [37, 38] and then multiplexed immunoassays such as addressable laser bead immunoassays (ALBIA) [39, 40], antigen arrays on planar surfaces [41-43], nanobarcodes [44] and chemiluminescence [45]. Such advances have been a boon to the modern diagnostic laboratory, because these platforms have facilitated automated, high throughput, increasingly inexpensive and rapid turnaround time test results.

In the mix of emerging technologies to detect aab and the widespread familiarity and reliance on IIF, it is clear that, depending on the assay used, the results that one can get from the new technologies can be at considerable variance. This has led to debates of the relative value of old and new diagnostic platforms where the implications of false-negative versus false-positive test results are being considered (reviewed in [46]). There has been a tendency to adhere to things that are easily understood, fit within existing diagnostic paradigms and seem to have immediate clinical relevance. Hence, some prefer IIF on specified substrates (i.e., HEp-2 and certain tissue sections) as the "gold standard" for autoantibody testing [47]—one of the associated claims being that this class of substrates contains well over 100 different target antigens, whereas newer screening technologies may be limited to $<20$ [48]. While that may be true, it is abundantly clear that IIF does not detect all aab even when they are directed to an autoantigen that is highly expressed in HEp-2 cells and otherwise reacts with the same aab that are found in other sera. For example, a significant proportion of sera that have antibodies directed against Jo-1 [49], ribosomal P proteins [50, 51], PCNA [25], GWB [52] and PM/Scl [53] (to name a few) are not detected by IIF on HEp-2 substrates. While the facile argument is that these 'false-negative' IIF results are likely attributable to autoantibody titers, hidden or cryptic epitopes, the IIF technique itself or characteristics of the substrate (i.e., cell density, growth media, fixation protocols), the evidence to support such conclusions is far from uniform or convincing. A case in point is aab to proteinase 3 (PR3), which are detected by conventional techniques such as ELISA and generally recognized as a cANCA IIF pattern on human neutrophil substrates. However, in a recent international study of sera from ulcerative colitis patients who typically show an atypical ANCA (aANCA or 
xANCA) IIF pattern and on a PR3 ELISA are negative; in a novel chemiluminescence assay using the identical PR3 antigen preparation (Bio Flash, INOVA Diagnostics, San Diego, CA, USA), many sera had remarkable anti-PR3 activity (manuscript in revision).

Rapid advances in diagnostic technologies have made it difficult for even the most modern laboratory to keep abreast, not to mention the clinicians who are hard pressed to adapt to and accept new diagnostic paradigms that are built on newer technologies. Before we can hope to completely understand the enigmas that attend results of aab diagnostic testing, perhaps it is time to step back from and re-examine long accepted paradigms and beliefs. This review will address some of the issues that impact on contemporary "golden age" technologies and some considerations that will be important as we move to "new age" platforms. This will be addressed in the context of aab that are "bad" (pathogenic), "good" (protective) or "indifferent" (no apparent role in disease). Finally, we will conclude by looking ahead to newer concepts and technologies and suggest that some old concepts upon which aab testing is based, as well as emerging ideas on origin and functions of aab, should be re-examined.

\section{Autoantibodies: the bad, good and indifferent}

It is clear that the terminology and concept of "horror autotoxicus" coined by Nobel Laureate Paul Ehrlich has had an indelible impact on our view of aab as being "bad" through presumed involvement in the genesis and pathogenesis of the SARD (reviewed in [54]). This view has been abetted by evidence that anti-acetylcholine receptor $\mathrm{aab}$ in myasthenia gravis were a key to the clinical expression and pathogenesis of the disease (reviewed in [55]) and more recent evidence that aab binding the water channel aquaporin 4 are pathogenic in neuromyelitis optica and transverse myelitis (reviewed in [56-58]). Accordingly, there has been a long and arduous search for evidence that aab in systemic lupus, systemic sclerosis, rheumatoid arthritis and other SARD are pathogenic [59]. Early evidence that aab directed to double-stranded DNA participate and/or initiate lupus nephritis has waxed and waned along with fairly compelling evidence that antibodies to the protein components of chromatin are possibly more directly related to the disease process (reviewed in [60]). Similarly, anti-DNA antibodies that cross-react with $N$-methyl-D-aspartate receptor (NMDR) have been demonstrated to be pathogenic and related to neuropsychiatric lupus [61, 62], although these observations have not been widely corroborated. Similarly, aab directed to platelet derived growth factor were promoted to contribute to a unifying paradigm in antibody-mediated pathogenesis of systemic sclerosis [63, 64], although other unpublished studies have yet to successfully corroborate that evidence (personal communication, Dr. M Servant, McGill University). The list could go on, but the point is despite what we might have been taught in introductory immunology, whereas the evidence supporting antibody-mediated pathogenesis in certain organ-specific autoimmune diseases is substantiated, the evidence supporting autoantibody-mediated disease in SARD is far from compelling.

Fortunately, the potential pathogenic role of aab is balanced by growing evidence that some aab are "good" or protective (reviewed in [65-67]). Indeed, the dogma that autoimmune processes lead to tissue damage has been refuted by a growing data in which autoimmune mechanisms in general, and aab specifically, have been demonstrated to be protective against disease genesis and processes. Even Paul Ehrlich himself was dispassionate about the notion of "horror autotoxicus" when he considered that anti-autotoxin antibodies might exist. However, as part of the contemporary immunology genre, Elie Metchnikoff and Alexander Besredka perpetuated the notion of autoimmunity and the notion that self-reactive antibodies could be controlled by anti-antibodies (reviewed in [68]).

To quote Arturo Casadevall, "the view that immunoglobulins function largely by potentiating neutralization, cytotoxicity or phagocytosis is being replaced by a new synthesis whereby antibodies participate in all aspects of the immune response, from protecting the host at the earliest time of encounter with a microbe to later challenges. Perhaps the most transformative concept is that immunoglobulins manifest emergent properties, from their structure and function as individual molecules to their interactions with microbial targets and the host immune system. Given that emergent properties are neither reducible to first principles nor predictable, there is a need for new conceptual approaches for understanding antibody function and mechanisms of antibody immunity" [69].

Evidence supporting the supposition that we all bear our own distinctive aab repertoire is supported by substantial evidence (reviewed in [68]). A recent excellent example is the observation that apparently healthy people harbor anti-PR3, myeloperoxidase and glomerular basement membrane type IV collagen antibodies [70]: aab that are generally characteristic of granulomatosis with polyangiitis (Wegener's syndrome) and related vasculopathies [71]. These so-called 'natural aab' tend to be polyreactive, primarily IgM isotype, and react with both self and non-self targets. It is thought that some of these relatively nonspecific and low-affinity binding natural aab may prevent 
autoreactive cells from binding self-antigens by masking their antigenic determinants. These and other studies suggest that SARD may be prevented or ameliorated by protective "good" aab and that dampening the B cell response through anti-CD20 and related therapies may not be the best therapeutic approach. Hence, the identification and utilization of protective aab may serve as a much more interesting and potentially useful paradigm for novel therapies than current approaches that target cytokines, their cognate receptors or other multi-functional receptors as an approach to the treatment of SARD and other autoimmune diseases.

While the therapeutic paradigm of cytokine and cytokine receptor and other immune reactant blockade has had remarkable success (i.e., anti-TNF), there are likely more practical and perhaps less toxic ways to treat SARD in the future. For example, the administration of IgM anti-dsDNA antibodies into SLE-prone mice prevented the development of nephritis and the presence of rheumatoid factor (RF) in SLE was suggested by some to be protective against the development of lupus nephritis (reviewed in [65]).

Whereas some aab may be considered "bad" (pathogenic) or "good" (protective), it must also be considered that certain aab in an individual's repertoire is "indifferent" - not related to pathogenesis or protection. This concept is supported by some studies in antibody responses to certain microorganisms where there is no apparent role for certain antibodies in isolation [69]. Perhaps reuniting with our "forefather" microbiologists and taking a systems biology approach to SARD and other autoimmune diseases is part of our desired future [72].

\section{Breaking down paradigms}

The forgoing discussion should serve as an impetus to all stakeholders in aab diagnostics (educators, clinicians, diagnostic laboratories, regulators and diagnostic kit manufacturers) to reconsider what they have been taught and have come to believe about aab. In today's world of diagnostics, overarching concerns about false-negative and false-positive aab tests that attend virtually all aab diagnostic platforms [46] should not be summarily dismissed by citing pros or cons of any one technology or diagnostic platform. Many of these issues point to persisting significant gaps in our understanding of the origin and roles of aab. Before we can hope to completely understand the enigmas that attend the results of aab diagnostic tests, perhaps it is time to step back and reexamine long accepted paradigms and beliefs. Some of the issues about aab that should be (re)considered in the future are discussed very briefly below and are outlined in Table 1.
Table 1 Future considerations of characteristics, functions and roles of autoantibodies that impact on their diagnostic relevance

- Role of autoantibodies in personalized medicine

- The host bearing the autoantibody

- The "trigger" that initiated the autoantibody response

- Autoantibody efficacy: a function of specificity, amount, isotype, host genetics/epigenetics

- Autoantibody isotypes and subclasses

- Reconsider the concept of "prozone"

- Autoantibody binding complement and/or other proteins

- Re-evaluate the potential importance of the autoantibody Fc

- Peptoid technology: define novel and disease relevant autoantigens and use them in diagnostics

- Sort the "wheat from the chaff"

First, while aab testing is traditionally considered to primarily serve the realm of diagnostic medicine, there is little doubt that aab testing is rapidly being recognized as a discipline that will provide important prognostic and therapeutic information at the bedside. For example, the European consortium (EUSTAR) studying more than 3,600 systemic sclerosis concluded that the "clinical distinction seemed to be superseded by an antibody-based classification" [73]. And a recent report suggested that an autoantibody-based classification of SLE has clinical value [74]. Accordingly, along with other proteomic analyses (i.e., cytokine profiles), genomics and metabolomics, aab testing is taking on increasing importance in the realm of personalized medicine [75].

Second, in terms of differentiating "good" from "bad" or "indifferent" aab, it needs to be appreciated that the terms 'protective' and 'non-protective' are relative terms that depend on a number of factors: the chief among them being the host and the "trigger" that initiated the response $[69,76]$. Indeed, inroads into our understanding of aab test results and aab functions will not be significantly advanced until they are understood in the context of the entire patient, and in particular any co-morbidities that may be present. Studies of clinical correlations of aab based only on simple diagnostic stratifications must take into account co-morbidities, which have an important influence both on the repertoire of aab produced and on the expression of autoimmune disease (i.e., disease phenotype). To achieve a more complete and meaningful serological profile, it will be particularly important to combine aab profiles with cytokine and other proteomic profiles in addition to genomics and metabolomics. While the amount of data generated in such studies can be overwhelming, bioinformatics is poised to permit such complex analyses and 'paint' a more comprehensive and realistic picture of clinical subsets of disease.

Third, the efficacy of aab must be considered. Based on primarily microbiological studies [69], the protective 
efficacy of antibodies has been shown to be a function of specificity, amount, genetic and epigenetic characteristics of the host and, as presented in the next paragraph, isotypes. As implied earlier in this review, the same applies to defining and understanding pathogenic or indifferent aab.

Fourth, the isotypes and subclasses of aab in any given patient are very important. There is ample evidence in the broad aab literature that certain aab, expressed as certain isotypes or subclasses, make a big difference in terms of diagnostic, therapeutic and, by extension, prognostic importance. Not that many years ago, most autoimmunologists probably did not care that much about IgG4. However, today the emergence of clinical syndromes based on IgG4 aab and immune responses [77, 78] should be cause for reconsidering the entire spectrum of diseases that are both conventional and non-conventional autoimmune diseases. For one thing, antibody isotypes are considered to point to the triggering pathogen. For example, in $\mathrm{C}$. neoformans infections IgG2a $>\operatorname{IgG} 1>\operatorname{IgG} 2 \mathrm{~b} \gg>\operatorname{IgG} 3$, whereas in Mycobacterium tuberculosis IgG3 $\gg$ IgG2 [69]. Such considerations in antigen or autoantigen driven or mediated $\mathrm{aab}$ responses may point to the elusive trigger(s) of autoimmunity.

Fifth, while the concept of "prozone" is generally thought of only in terms of in vivo or solution phenomena, this needs to be reconsidered and explored in the context of aab detection in both older and newer diagnostic platforms $[79,80]$. Not all diagnostic platforms have the same antigen density available for aab binding or the same dynamic range. Newer platforms such as BioFlash that employ a bead-based chemiluminescence technology appear to provide advantages in this regard [45].

A sixth consideration is to determine if the aab in question binds complement and other proteins. A recent report indicating that synovial fluid complement, specifically, the membrane attack complex-mediated arm of complement, is crucial to the development of joint disease in three different models of osteoarthritis [81] may come as a surprise to some autoimmunologists, but it does highlight the importance of re-evaluating the complement-binding and complement-activating capacity of not only aab, but other proteins (i.e., integrins) involved in immune mediate diseases. Such studies open up connections between adaptive immunity with numerous cellular components such as matrix metalloproteases (MMPs), extracellular signal-regulated kinase (ERKs) and integrins. Recent evidence has provided renewed interest in complement as being a critical feature of ANCA-related vasculitis [82], anti-aquaporin 4-related neuromyelitis optica [83] and models of aab-induced hepatitis [84].

Seventh, while much attention has focused on the Fabidiotype of $a a b$, it is time to reconsider the potential importance of the Fc. There is now convincing evidence that the Fc component of antibodies has a dramatic effect on the reactivity of the Fab insomuch as Fc influences the fine specificity of antibody reactivity (reviewed in [69]). Contemporary views that aab are simply bifunctional molecules composed of independent Fab and Fc domains is no longer tenable, since it has been clearly shown that the $\mathrm{V}$ region of $\mathrm{Fab}$ and the $\mathrm{C}$ region of $\mathrm{Fc}$ act together to affect both affinity and specificity of antibody binding [85]. In part, this effect has also been attributed to observations that IgG Fc receptors have both stimulatory and inhibitory effects on cells [69].

Eighth, based on a lesson that could be learned from a major advance in the diagnosis of rheumatoid arthritis (RA), with the discovery of aab to citrullinated peptides by van Venrooij and his colleagues (reviewed in [86]), is the importance of autoantigens and analytes for aab detection that are related to the disease itself. In the past, approaches to identifying target autoantigens has had a modicum of success and in some cases major breakthroughs using conventional technologies employed by cell and molecular biologists. These have included immunoprecipitation of the target antigens followed by molecular analysis (i.e., mass spectroscopy), immunoscreening of recombinant proteins produced by expression clones and immunoscreening arrays of native and recombinant proteins and peptide arrays absorbed or 'printed' on solid phase substrates such as nitrocellulose or glass. The basic protocol for the latter included pouring index patient sera containing diseasespecific antibodies of potential interest over the array and then seeing what sticks. The results were then compared to "controls" of an unrelated disease and normal persons, and "voilà" you had your new candidate target autoantigen and biomarker for the disease of interest. In a related novel approach, Kodadek and his colleagues at the Scripps Research Institute in Jupiter, Florida (USA) have reasoned that it is unlikely that aab from a person with a given disease would primarily bind to "normal" proteins [87]. In this paradigm, it is suggested that the immune system does not react to normal proteins simply because 'tolerance to normal proteins has been broken'. Indeed, it seems more intuitive that the immune system reacts to foreign proteins of an invading microorganism or proteins altered in dying (i.e., senescent, apoptotic or necrotic) cells. Even more intriguing is the possibility that the target antigens are released from living cells as extracellular exosomes or microbodies [88, 89] during disease genesis when certain targets are altered either because of epigenetic effects, genetic mutations, microRNA regulation, proteasomal or exosomal dysregulation or simply post-translational modifications (i.e., citrullination) of the targets due to microenvironmental changes. Because the potential by-products of all of these events are logistically overwhelming, instead of using arrays of normal proteins, Kodadek and his 
colleagues employ a fairly random assortment of unrelated synthetic small molecules that bind to aab because of their chemical shapes (epitopes), allowing for a very broad and unbiased "fishing trip". The difference from earlier "fishing trips" that used large peptide/protein arrays is the "bait" (also referred to as 'peptoids') that is used [90]. So, nice idea, but does it work? As one early example, this approach has yielded novel aab targets in Alzheimer's disease [91]. Obviously, there is much more to be learned about this approach, not to mention the corroborated evidence of success, but it seems likely that peptoid chemistry will be a major advancement in the field of autoimmune diagnostics and therapeutics.

Ninth, if it is true that some aab are pathogenic and some are actually "good for you" or protective, and some may have less intuitive functions, then it is very important to "sort the wheat from the chaff" (quotation: Dr. Ian McKay). This is particularly critical in the future when aab detection not only will be more than a tool for early and accurate diagnosis, but also will provide key information about an individual patient's aab mosaic that reflects good, bad and, presumably, indifferent antibodies.

In summary, it appears that aab testing is on the threshold of new applications that extends beyond diagnostics into the realm of prognostics, therapeutics and personalized medicine. However, to move into this "new age", a much clearer and more thorough understanding of the genesis and roles of aab is required. This also includes appreciating that the two solitudes of innate B cell immunity and acquired immunity are a continuum, and this is attended by re-examining old paradigms and adopting new technological approaches to arrive at a new aab orthodoxy.

Conflict of interest None.

\section{References}

1. Ehrlich P (1900) On immunity with special reference to cell life. Proc R Soc Lond 66:424-448

2. Wassermann VA, Neisser A, Bruck C (1906) Eine serodiagnostische reaktion bei syphilis. Deutsche Medicinische Wochenshrift 19:745-746

3. Moore JE, Mohr CF (1952) Biologically false positive serologic tests for syphilis. J Am Med Assoc 150:467-473

4. Tuffanelli DL (1968) False-positive reactions for syphilis. Serological abnormalities in relatives of chronic reactors. Arch Derm 98:606-611

5. Koike T, Sueishi M, Funaki H, Tomioka H, Yoshida S (1984) Anti-phospholipid antibodies and biological false positive serological test for syphilis in patients with systemic lupus erythematosus. Clin Exp Immunol 56:193-199

6. Conrad K, Schlosser W, Hiepe F, Fritzler MJ (2011) Autoantibodies in organ specific autoimmune diseases: a diagnostic reference. Pabst Science, Lengerich
7. Hargraves MM, Richmond H, Morton R (1948) Presentation of two bone marrow elements: the "tart" cells and the "L.E." cell. Mayo Clin Proc 23:25-28

8. Conn RB (1994) Practice parameter: the lupus erythematosus cell test. Am J Clin Pathol 101:65-66

9. Fritzler MJ (1986) Autoantibody testing: procedures and significance in systemic rheumatic diseases. Meth Achiev Exp Pathol $12: 224-260$

10. Warde N (2011) Connective tissue diseases: agonistic autoantibodies: do they have a role in the pathophysiology of SSc? Nat Rev Rheumatol 7:71

11. Weller T, Coons AH (1954) Fluorescent antibody studies with agents of varicella and herpes zoster propagated in vitro. Proc Soc Exp Biol Med 86:789-794

12. Fritzler MJ, Wiik A (2006) Autoantibody assays, testing, and standardization. In: Rose NR, Mackay IR (eds) The autoimmune diseases, 4th edn. Elsevier Academic Press, Sydney, pp 10111022

13. Holborow EJ, Weir DM, Johnson GD (1957) A serum factor in lupus erythematosus with affinity for tissue nuclei. Br Med $\mathrm{J}$ 2:732-734

14. Hijmans W, Schuit HRE, Mandema E et al (1964) Comparative study for the detection of antinuclear factors with the fluorescent antibody technique. Ann Rheum Dis 23:73-77

15. Kunkel HG, Tan EM (1964) Autoantibodies and disease. Adv Immunol 4:351-372

16. Beck JS (1961) Variations in the morphological patterns of "autoimmune" nuclear fluorescence. Lancet 1:1203-1207

17. Nakamura RM, Tan EM (1977) Recent progress in the study of autoantibodies to nuclear antigens. Human Pathol 9:85-91

18. Nakamura RM, Peebles CL, Molden DP, Tan EM (1984) Advances in laboratory tests for autoantibodies to nuclear antigens in systemic rheumatic diseases. Lab Med 15:190-198

19. Lucey BP, Nelson-Rees WA, Hutchins GM (2009) Henrietta Lacks, HeLa cells, and cell culture contamination. Arch Pathol Lab Med 133:1463-1467

20. Nelson-Rees WA, Daniels DW, Flandermeyer RR (1981) Crosscontamination of cells in culture. Science 212:446-452

21. Tan EM, Feltkamp TEW, Smolen JS et al (1997) Range of antinuclear antibodies in "healthy individuals. Arthr Rheum 40:1601-1611

22. Sherer Y, Gorstein A, Fritzler MJ, Shoenfeld Y (2004) Autoantibody explosion in systemic lupus erythematosus. Semin Arthr Rheum 34:501-537

23. Sherer Y, Shoenfeld Y (2007) Autoantibody explosion in lupus155 different autoantibodies in SLE. Lupus 16(suppl):42

24. Walker JG, Fritzler MJ (2007) Update on autoantibodies in systemic sclerosis. Curr Opin Rheumatol 19:580-591

25. Mahler M, Silverman ED, Fritzler M (2010) Novel diagnostic and clinical aspects of anti-PCNA antibodies detected by novel detection methods. Lupus 19:1527-1533

26. Fritzler MJ, Rattner JB, Luft LM et al (2010) Historical perspectives on the discovery and elucidation of autoantibodies to centromere proteins (CENP) and the emerging importance of antibodies to CENP-F. Autoimmun Rev 10:194-200

27. Reimer G, Raska I, Tan EM, Scheer U (1987) Human autoantibodies: probes for nucleolus structure and function. Virchows Arch B 54:131-143

28. Welting TJ, Raijmakers R, Pruijn GJ (2003) Autoantigenicity of nucleolar complexes. Autoimmun Rev 2:313-321

29. Enarson P, Rattner JB, Ou Y, Miyachi K, Horigome T, Fritzler MJ (2004) Autoantigens of the nuclear pore complex. J Mol Med 82:423-433

30. Fritzler MJ, Stinton LM, Chan EKL (2007) Autoantibodies to cytoplasmic autoantigens in endosomes, exosomes and the Golgi complex. In: Conrad K, Chan EKL, Fritzler MJ, Sack U, 
Shoenfeld Y, Wiik A (eds) From etiopathogenesis to the prediction of autoimmune diseases: relevance of autoantibodies, 5th edn. Pabst Science, Lengerich, pp 194-209

31. Tan EM (1989) Antinuclear antibodies: diagnostic markers for autoimmune diseases and probes for cell biology. Adv Immunol 44:93-151

32. Halbert SP, Karsh J, Anken M (1981) Studies on autoantibodies to deoxyribonucleic acid and deoxyribonucleoprotein with enzyme-immunoassay (ELISA). J Lab Clin Med 97:97-111

33. Reichlin M, Harley JB (1986) Detection by ELISA of antibodies to small RNA protein particles in SLE patients whose sera lack precipitins. Trans Assoc Am Phys 99:161-171

34. Tonutti E, Bassetti D, Piazza A et al (2004) Diagnostic accuracy of ELISA methods as an alternative screening test to indirect immunofluorescence for the detection of antinuclear antibodies. Evaluation of five commercial kits. Autoimmunity 37:171-176

35. Stott DI (1989) Immunoblotting and dot blotting. J Immunol Methods 119:153-187

36. Nezlin R, Mozes E (1995) Detection of antigens in immune complexes by a dot blot assay. J Immunol Methods 184:273-276

37. Damoiseaux J, Boesten K, Giesen J, Austen J, Tervaert JW (2005) Evaluation of a novel line-blot immunoassay for the detection of antibodies to extractable nuclear antigens. Ann NY Acad Sci 1050:340-347

38. Pottel H, Wiik A, Locht $\mathrm{H}$ et al (2004) Clinical optimization and multicenter validation of antigen-specific cut-off values on the INNO-LIA ANA update for the detection of autoantibodies in connective tissue disorders. Clin Exp Rheumatol 22:579-588

39. Fritzler MJ (2006) Advances and applications of multiplexed diagnostic technologies in autoimmune diseases. Lupus $15: 422-427$

40. Fritzler MJ, Fritzler ML (2009) Microbead-based technologies in diagnostic autoantibody detection. Expert Opin Med Diagn 3:81-89

41. Baker CA, Lu ZY, Manuelidis L (2004) Early induction of interferon-responsive mRNAs in Creutzfeldt-Jakob disease. J Neurovirol 10:29-40

42. Balboni I, Limb C, Tenenbaum JD, Utz PJ (2008) Evaluation of microarray surfaces and arraying parameters for autoantibody profiling. Proteomics 8:3443-3449

43. Chandra PE, Sokolove J, Hipp BG et al (2011) Novel multiplex technology for diagnostic characterization of rheumatoid arthritis. Arthr Res Ther 13:R102

44. Freeman RG, Raju PA, Norton SM et al (2005) Use of nanobarcodes particles in bioassays. Methods Mol Biol 303:73-83

45. Mahler M, Radice A, Sinico RA et al (2011) Performance evaluation of a novel chemiluminescence assay for detection of antiGBM antibodies: an international multicenter study. Nephrol Dial Transplant 27:243-252

46. Fritzler MJ (2011) The antinuclear antibody (ANA) test: last or lasting gasp? Arthr Rheum 16:19-22

47. Meroni PL, Schur PH (2010) ANA screening: an old test with new recommendations. Ann Rheum Dis 69:1420-1422

48. American College of Rheumatology (2011) Position paper: methodology of testing for antinuclear antibodies

49. Hoffman IE, Peene I, Veys EM, De KF (2002) Detection of specific antinuclear reactivities in patients with negative antinuclear antibody immunofluorescence screening tests. Clin Chem 48:2171-2176

50. Mahler M, Kessenbrock K, Raats J, Fritzler MJ (2004) Technical and clinical evaluation of anti-ribosomal $\mathrm{P}$ protein immunoassays. J Clin Lab Anal 18:215-223

51. Mahler M, Ngo J, Schulte-Pelkum J, Luettich T, Fritzler MJ (2008) Limited reliability of the indirect immunofluorescence technique for the detection of anti-Rib-P antibodies. Arthr Res Ther 10:R131
52. Stinton LM, Swain M, Myers RP, Shaheen AA, Fritzler MJ (2010) Autoantibodies to GW bodies and other autoantigens in primary biliary cirrhosis. Clin Exp Immunol 163:147-156

53. Mahler M, Fritzler MJ (2009) The changing landscape of the clinical value of the PM/Scl autoantibody system. Arthr Res Ther $11: 106$

54. Elkon K, Casali P (2008) Nature and functions of autoantibodies. Nat Clin Pract Rheumatol 4:491-498

55. de Baets MH (2010) Insights in the autoimmunity of myasthenia gravis. Autoimmunity 43:341-343

56. Saikali P, Cayrol R, Vincent T (2009) Anti-aquaporin-4 autoantibodies orchestrate the pathogenesis in neuromyelitis optica. Autoimmun Rev 9:132-135

57. Cayrol R, Saikali P, Vincent T (2009) Effector functions of antiaquaporin-4 autoantibodies in neuromyelitis optica. Ann NY Acad Sci 1173:478-486

58. Benavente E, Paira S (2011) Neuromyelitis Optica-AQP4: an update. Curr Rheumatol Rep 13:496-505

59. Mackay IR, Leskovsek NV, Rose NR (2008) Cell damage and autoimmunity: a critical appraisal. J Autoimmun 30:5-11

60. van der Vlag J, Berden JH (2011) Lupus nephritis: role of antinucleosome autoantibodies. Semin Nephrol 31:376-389

61. Scheinecker C, Bonelli M, Smolen JS (2010) Pathogenetic aspects of systemic lupus erythematosus with an emphasis on regulatory T cells. J Autoimmun 35:269-275

62. Faust TW, Chang EH, Kowal C et al (2010) Neurotoxic lupus autoantibodies alter brain function through two distinct mechanisms. Proc Natl Acad Sci USA 107:18569-18574

63. Tan FK (2006) Autoantibodies against PDGF receptor in scleroderma. N Engl J Med 354:2709-2711

64. Gabrielli A, Svegliati S, Moroncini G, Luchetti M, Tonnini C, Avvedimento EV (2007) Stimulatory autoantibodies to the PDGF receptor: a link to fibrosis in scleroderma and a pathway for novel therapeutic targets. Autoimmun Rev 7:121-126

65. Shoenfeld Y, Toubi E (2005) Protective autoantibodies: role in homeostasis, clinical importance, and therapeutic potential. Arthr Rheum 52:2599-2606

66. Meroni PL, Shoenfeld Y (2008) Predictive, protective, orphan autoantibodies: the example of the anti-phospholipid antibodies. Autoimmun Rev 7:585-587

67. Toubi E, Shoenfeld Y (2007) Protective autoimmunity in cancer (Review). Oncol Rep 17:245-251

68. Jennette JC, Falk RJ (2010) The rise and fall of horror autotoxicus and forbidden clones. Kidney Int 78:533-535

69. Casadevall A, Pirofski LA (2011) A new synthesis for antibodymediated immunity. Nat Immunol 13:21-28

70. Cui Z, Zhao MH, Segelmark M, Hellmark T (2010) Natural autoantibodies to myeloperoxidase, proteinase 3, and the glomerular basement membrane are present in normal individuals. Kidney Int 78:590-597

71. Jennette JC (2011) Nomenclature and classification of vasculitis: lessons learned from granulomatosis with polyangiitis (Wegener's granulomatosis). Clin Exp Immunol 164(Suppl 1):7-10

72. Biron CA, Casadevall A (2010) On immunologists and microbiologists: ground zero in the battle for interdisciplinary knowledge. MBio 1. (pii: e00260-10)

73. Walker UA, Tyndall A, Czirjak L et al (2007) Clinical risk assessment of organ manifestations in systemic sclerosis-a report from the EULAR scleroderma trials and research (EUSTAR) group data base. Ann Rheum Dis 66:754-763

74. Ching KH, Burbelo PD, Tipton C et al (2012) Two major autoantibody clusters in systemic lupus erythematosus. PLoS ONE 7:e32001

75. Fritzler MJ (2011) Personalized medicine approaches in rheumatoid arthritis and other systemic autoimmune rheumatic diseases. In: Conrad K, Chan EKL, Fritzler MJ, Humbel RL, Meroni 
PL, Shoenfeld Y (eds) From prediction to prevention of autoimmune diseases. Pabst Science, Berlin, pp 127-137

76. Pirofski LA, Casadevall A (2012) Q and A what is a pathogen? A question that begs the point. BMC Biol 10:6

77. Nirula A, Glaser SM, Kalled SL, Taylor FR (2011) What is IgG4? A review of the biology of a unique immunoglobulin subtype. Curr Opin Rheumatol 23:119-124

78. Stone JH, Zen Y, Deshpande V (2012) IgG4-related disease. N Engl J Med 366:539-551

79. Dubois-Galopin F, Beauvillain C, Dubois D et al (2007) New markers and an old phenomenon: prozone effect disturbing detection of filaggrin (keratin) autoantibodies. Ann Rheum Dis 66:1121-1122

80. Schnaidt M, Weinstock C, Jurisic M, Schmid-Horch B, Ender A, Wernet D (2011) HLA antibody specification using single-antigen beads-a technical solution for the prozone effect. Transplantation 92:510-515

81. Wang Q, Rozelle AL, Lepus CM et al (2011) Identification of a central role for complement in osteoarthritis. Nat Med 17:1674-1679

82. Kallenberg CG (2011) Pathogenesis of ANCA-associated vasculitis, an update. Clin Rev Allergy Immunol 41:224-231

83. Tradtrantip L, Zhang H, Anderson MO et al (2012) Small-molecule inhibitors of NMO-IgG binding to aquaporin-4 reduce astrocyte cytotoxicity in neuromyelitis optica. FASEB J
84. Tu Z, Li Q, Chou HS et al (2011) Complement mediated hepatocytes injury in a model of autoantibody induced hepatitis. Immunobiology 216:528-534

85. Torres M, Casadevall A (2008) The immunoglobulin constant region contributes to affinity and specificity. Trends Immunol 29:91-97

86. Van Venrooij WJ, van Beers JJ, Pruijn GJ (2011) Anti-CCP antibodies: the past, the present and the future. Nat Rev Rheumatol 7:391-398

87. Astle JM, Simpson LS, Huang Y et al (2010) Seamless bead to microarray screening: rapid identification of the highest affinity protein ligands from large combinatorial libraries. Chem Biol 17:38-45

88. Ullal AJ, Reich CF III, Clowse M et al (2011) Microparticles as antigenic targets of antibodies to DNA and nucleosomes in systemic lupus erythematosus. J Autoimmun 36:173-180

89. Pisetsky DS (2012) Microparticles as autoantigens: making immune complexes big. Arthr Rheum

90. Lindstrom TM, Robinson WH (2011) Fishing for biomarkers with antigen mimics. Cell 144:13-15

91. Reddy MM, Wilson R, Wilson J et al (2011) Identification of candidate IgG biomarkers for Alzheimer's disease via combinatorial library screening. Cell 144:132-142 